\title{
DARP-36aa Selectively Promotes Survival and Morphological Development of Cultured Mesencephalic Neurons
}

\author{
Sean M. Smith and Victor D. Ramirez \\ Neuroscience Program, Department of Molecular and Integrative Physiology, University of Illinois at Urbana-Champaign, Urbana, Illinois 61801
}

We examined the effects of DARP-36aa on the survival and morphological development of embryonic rat mesencephalic neurons. Treatment of mesencephalic cultures with DARP-36aa, a synthetic peptide corresponding to the N terminal of dopamine-releasing protein (DARP), resulted in a 1.8-fold increase in neuron survival. Morphological analysis revealed that DARP-36aa-treated neurons contained $48 \%$ more branching points per neuron compared with controls. DARP-36aa selectively affected mesencephalic cultures; diencephalic and C6 glioma cells were not affected by DARP-36aa treatments. Mesencephalic cultures were also incubated with polyclonal antibodies against DARP-36aa (anti-DARP-36aa) to assess the effect of immunoneutralization of endogenous DARP on these cells. Mesencephalic cultures treated with anti-DARP-36aa contained 43\% fewer neurons, and the number of branching points per neuron was decreased by nearly twofold compared with cultures grown with medium alone. Similar to cultures treated with DARP-36aa, immunoneutralization of DARP had no effect on any parameters examined in primary diencephalic and C6 glioma cultures. Mesencephalic cultures maintained in the presence of DARP-36aa had a 3.2-fold increase in the number of tyrosine hydroxylase (TH)-immunoreactive neurons, whereas anti-DARP-36aa incubations decreased TH-immunoreactive neurons by $40 \%$ compared with control cultures. Finally, coincubation of the specific tyrosine kinase inhibitor genistein with DARP-36aa resulted in a complete attenuation of DARP-36aamediated neuron survival and development in mesencephalic cultures. The findings indicate that DARP-36aa is a novel neurotrophic peptide that selectively promotes the survival and development of mesencephalic neurons.

Key words: DARP-36aa; neurotrophic factor; mesencephalon; tyrosine hydroxylase; catecholamine; diencephalon

\section{Introduction}

Neurotrophic factors regulate the survival and differentiation of developing neurons as well as maintain and promote the regeneration of mature neurons (Barde, 1989; Lindsay et al., 1994; Mufson et al., 1999). Neurotrophic factors are widely expressed and regulate diverse populations of neurons throughout the CNS (Kokaia et al., 1993; Gibbs and Pfaff, 1994; Lindsay et al., 1994; Mufson et al., 1999). In particular, characterization of the trophic actions of glial cell line-derived neurotrophic factor (GDNF) on the mesencephalic dopaminergic system has been well studied. GDNF stimulation results in increased cell size, neurite extension, and expression of phenotypic markers in dopaminergic neurons (Lapchak et al., 1996; Clarkson et al., 1997; Grondin and Gash, 1998). However, the survival of most populations of neurons depends not only on a single survival factor but also on several factors from multiple families of known and yet undefined trophic factors (Korsching, 1993; Snider, 1994).

Dopamine (DA)-releasing protein (DARP) was originally purified from the rat adrenal gland and shown to rapidly induce the release of DA from in vitro striatal tissue (Chang and Ramirez,

Received June 25, 2002; revised 0ct. 8, 2002; accepted 0ct. 18, 2002.

This work was supported in part by a grant from the National Institutes of Health (NIH) to V.D.R. and by an NIH Systems and Integrative Biology Training Grant fellowship to S.M.S. We thank Dr. Charles L. Cox for the use of his imaging equipment and Elizabeth A. Ainsworth for help with the preparation of this manuscript.

Correspondence should be addressed to Dr. Victor D. Ramirez, University of Illinois, 524 Burrill Hall, Department of Molecular and Integrative Physiology, 407 South Goodwin Avenue, Urbana, IL 61801. E-mail: vdramire@staff.uiuc.edu.

Copyright $\odot 2002$ Society for Neuroscience $\quad 0270-6474 / 02 / 220252-08 \$ 15.00 / 0$
1988). DARP immunoreactivity has been localized in both neurons and astrocytes in rat mesencephalic cell cultures as well as in C6 glioma cells, where it was secreted during culture (Smith and Ramirez, 1999). Kuhananthan et al. (1991) reported that the injection of DARP antibodies resulted in a marked increase in fetal resorption and a decrease in DA concentration in the midbrain of postnatal rats. Later, DARP was purified from the rat mesencephalon on embryonic day 17 (E17). Immunoneutralization of DARP on E17 significantly altered the DA concentration in the mesencephalon, whereas changes in DA levels were not detected in the diencephalon and telencephalon, suggesting that DARP may play a selective role in the development of dopaminergic neurons in the mesencephalon (Llano and Ramirez, 1994).

Immunopurification of DARP from primary mesencephalic cell cultures revealed that DARP is a multisubunit protein of 200 $\mathrm{kDa}$. Reduction of this $200 \mathrm{kDa}$ protein results in the generation of three subunits of $\sim 60,50$, and $25 \mathrm{kDa}$ (Ramirez and Marcus, 1992; patent number $5,146,786)$. N-terminal sequence information from purified DARP indicated that DARP has little sequence similarity with known neurotrophic factors. However, the first 33 aa of DARP were found to have $68 \%$ identity with serum albumin (Smith and Ramirez, 2002). Sequence information from immunopurified DARP was used in the synthesis of DARP-36aa, a synthetic 36 aa peptide. DARP-36aa induced DA release from in vitro striatal tissue at nanomolar concentrations (Ramirez and Marcus; patent number 5,146,786); antibodies generated against DARP-36aa had strong immunoreactivity with endogenous DARP (Smith and Ramirez, 1999). We have demonstrated re- 
cently that DARP-36aa enters C6 glioma and mesencephalic cells through receptor-mediated endocytosis pathways (Smith and Ramirez, 2002).

The aim of the present study was to expand on the findings that DARP selectively affects the survival and development of mesencephalic neurons. Using immunocytochemistry and stereological analysis, we investigated the effects of both DARP-36aa administration and DARP immunoneutralization on rat mesencephalic neurons, diencephalic neurons, and C6 glioma cells. We also investigated the neurotrophic properties of DARP-36aa by examining the effects of coincubation of genistein, a tyrosine kinase inhibitor, with DARP-36aa on mesencephalic neuron survival.

\section{Materials and Methods}

DARP-36aa peptide. Sequence information from the first 36 aa of the $\mathrm{N}$ terminal of the $60 \mathrm{kDa}$ subunit of immunopurified DARP (Smith and Ramirez, 2002) was used to synthesize DARP-36aa. The amino acid composition of DARP-36aa is DFHKSEIAHRFNDLGEKMFKMLNLDMRNMYLQQKTS.

Primary cell culture. Primary mesencephalic and diencephalic cell cultures were prepared from E17 rat pups (Sprague Dawley). Dissection of the diencephalon and mesencephalon was performed with the ventral aspect of the brain facing up. Diencephalic tissue was taken from the level of the optic chiasm through the posterior border of the hypothalamus. Mesencephalic tissue was removed from the posterior border of the hypothalamus to the anterior border of the pons. Mesencephalic and diencephalic tissues were placed into sterile Petri dishes containing $\mathrm{Mg}^{2+}$ / $\mathrm{Ca}^{2+}$-free Tyrode's solution. After dissection, tissue was diced into 1-3 $\mathrm{mm}$ cubes and washed twice with $20 \mathrm{vol}$ of $\mathrm{Mg}^{2+} / \mathrm{Ca}^{2+}$-free Tyrode's solution. Cells were pelleted by centrifugation at $325 \times g$ for $5 \mathrm{~min}$. The resulting pellet was incubated in $2 \mathrm{ml}$ of trypsin/EDTA (Sigma, St. Louis, $\mathrm{MO})$ at $37^{\circ} \mathrm{C}$ for $15 \mathrm{~min}$. Enzymatic digestion was stopped with the addition of a $2 \times$ vol of DMEM (Invitrogen, Gaithersburg, MD) containing $10 \%$ fetal bovine serum (FBS; Sigma). Tissue was then mechanically triturated using a sterile Pasteur pipette attached to a Pi-Pump (20 passes; Drummond Scientific, Broomall, PA). Cell suspensions in DMEM containing 10\% FBS were seeded onto poly-L-lysine/laminin-coated coverslips (Fisher, Pittsburgh, PA) in 24 well culture plates $\left(1.5 \times 10^{5}\right.$ cells/ well). Cultures were maintained at $37^{\circ} \mathrm{C}$ in a humidified atmosphere containing $94 \% \mathrm{O}_{2}$ and $6 \% \mathrm{CO}_{2}$.

C6 glioma cell cultures. C6 glioma cells (Benda et al., 1968) (American Type Culture Collection, Manassas, VA) were cultured on glass coverslips in 24 well culture plates (Corning, Acton, MA). Cells were plated at a density of $1.5 \times 10^{5}$ cells/well in Ham's F-12K medium (Invitrogen) supplemented with L-glutamine $(2 \mathrm{~mm})$, sodium bicarbonate $(1.5 \mathrm{gm} / \mathrm{l})$, $15 \%$ horse serum (Sigma), and $2.5 \%$ FBS. Cultures were kept at $37^{\circ} \mathrm{C}$ in a humidified atmosphere containing $94 \% \mathrm{O}_{2}$ and $6 \% \mathrm{CO}_{2}$.

Culture treatments. Primary mesencephalic, diencephalic, and C6 glioma cell cultures were processed in the same manner. Three independent experiments were performed with at least three culture wells examined per treatment group. Cultures treated with DARP-36aa were allowed to adhere overnight in serum-supplemented DMEM (primary cultures) or Ham's F-12K (C6 glioma cultures) culture medium. After adherence, the cells were washed three times and cultured in a defined medium consisting of Ham's F-12K and DMEM (1:1 ratio) with N2 supplements (Bottenstein et al., 1979) in the presence of 10 nм DARP-36aa, 50 nм DARP36aa, or N2 medium alone (control). Culture medium containing DARP-36aa was subsequently changed every other day until in vitro day (IVD) 7. Anti-DARP-36aa-treated cultures were grown for $8 \mathrm{~d}$ in serumsupplemented DMEM or Ham's F-12K medium. Cultures were incubated with either $10 \mu \mathrm{g}$ of anti-DARP-36aa or $50 \mu \mathrm{g}$ of anti-DARP-36aa on IVD 5 and IVD 7 and compared with control cultures grown in serum-supplemented medium alone. The tyrosine kinase inhibitor genistein (Sigma) was included in select primary mesencephalic culture incubations. Cultures received $10 \mathrm{~nm}$ DARP-36aa, $10 \mu \mathrm{g}$ of genistein, both $10 \mu \mathrm{g}$ of genistein and $10 \mathrm{~nm}$ DARP-36aa, or ethyl alcohol (EtOH; genistein vehicle) in serum-free N2 medium. Incubations were performed using the same procedure detailed above for DARP-36aa.

Immunocytochemistry. Primary mesencephalic cultures were processed for immunocytochemistry on IVD 7 (DARP-36aa treated) or IVD 8 (anti-DARP-36aa treated). Cells were washed with $0.1 \mathrm{~m}$ PBS and fixed for $30 \mathrm{~min}$ in $4 \%$ paraformaldehyde in $0.1 \mathrm{M}$ PBS. Cultures were blocked and permeabilized by a $1 \mathrm{hr}$ incubation in $0.1 \mathrm{M}$ PBS with $0.4 \%$ Triton $\mathrm{X}-100,1 \%$ bovine serum albumin (BSA), and $4 \%$ goat serum (GS). Subsequently, cells were incubated overnight at $4^{\circ} \mathrm{C}$ with anti-neuronspecific enolase (anti-NSE, 1:500; Chemicon, Temecula, CA) or antityrosine hydroxylase (anti-TH, 1:1000; Chemicon), in 0.1 M PBS with $0.4 \%$ Triton X-100, $1 \%$ BSA, and $1 \%$ GS. After primary antibody treatment, anti-rabbit IgG conjugated to horseradish peroxidase (1:300; Sigma) in PBS with $1 \%$ BSA was applied for $2 \mathrm{hr}$ at room temperature. Visualization of immunoreactive proteins was accomplished through the use of a 3,3'-diaminobenzidine (Sigma) substrate system. Staining specificity was determined by the substitution of $2 \%$ GS in $0.1 \mathrm{~m}$ PBS for the primary antibody.

Stereological analysis of cell cultures. The quantitative analysis of neuron number and cell morphology was accomplished using a field sampling technique described by Ventimiglia et al. (1995). Briefly, photomicrographs ( $40 \times$ objective) of a series of randomly selected populations in each culture well were taken, with six wells sampled per treatment group. Photomicrographs were enlarged until a final magnification of $800 \times$ was obtained, corresponding to a $5 \times 3 \mathrm{~mm}$ area of the culture well. A series of counting grids were superimposed onto the photomicrographs and used to determine neuron number, cell body area, and number of branch points for NSE-positive and TH-positive neurons.

Quantification of neuron and TH-positive cell number was determined using the following formula: $N_{\mathrm{t}}=(\Sigma N)(5 \mathrm{~mm})(3 \mathrm{~mm})\left(M^{2}\right) / A_{\mathrm{f}}$, where $N_{\mathrm{t}}$ is the total number of cells, $\Sigma \mathrm{N}$ corresponds to the sum of neurons counted in each photomicrograph, $(5 \mathrm{~mm})(3 \mathrm{~mm})$ is the area of the culture represented by the photograph, $M^{2}$ is the final magnification, and the total area of the counting frame (photograph) is represented by $A_{\mathrm{f}}$ (Ventimiglia et al., 1995b).

A second grid system, described by Ventimiglia et al. (1995), was used to determine the cell body area. The total number of grid points $(P)$ that fell within the cell body of each cell was multiplied by the total area represented by each point $\left(A_{\mathrm{p}}\right)$. The resulting value was corrected for total magnification $\left(M^{2}\right)$ : cell body area $=(P)\left(A_{\mathrm{p}}\right) / M^{2}$. A third grid system consisting of 35 small counting frames (frame area, $1 \mathrm{~cm}^{2}$ ) was superimposed onto each photomicrograph to determine the total number of neuronal branch points per culture well. The total number of branch points counted using the grid system was corrected for sampling frequency and magnification by the following formula: total branch point per well $=\left(\Sigma P_{\mathrm{B}}\right)(5 \mathrm{~mm})(3 \mathrm{~mm})\left(M^{2}\right) / A_{\mathrm{g}}$, where $\Sigma P_{\mathrm{B}}$ represents the sum of all branch points counted per photomicrograph and $A_{\mathrm{g}}$ is the total area of the grid system ( 35 frames $\times 1 \mathrm{~cm}^{2}$ ). The total number of branch points per well was then divided by the total number of neurons in the culture well to yield the number of branch points per neuron (Ventimiglia et al., 1995b).

Table 1. Effects of DARP-36aa and anti-DARP-36aa on mesencephalic neurons

\begin{tabular}{llcl}
\hline Treatment & $\begin{array}{l}\text { Neuron } \\
\text { number/well }\end{array}$ & $\begin{array}{l}\text { Cell body } \\
\text { area }\left(\mu \mathrm{m}^{2}\right)\end{array}$ & $\begin{array}{l}\text { Branching } \\
\text { points/neuron }\end{array}$ \\
\hline N2 control & $4.2 \pm 0.4 \times 10^{4}$ & $103 \pm 3.0$ & $5.6 \pm 0.3$ \\
$10 \mathrm{~nm}$ DARP-36aa & $7.8 \pm 0.6 \times 10^{4 * *}$ & $99 \pm 3.0$ & $8.3 \pm 0.6^{*}$ \\
$50 \mathrm{~nm}$ DARP-36aa & $7.2 \pm 1.0 \times 10^{4 * *}$ & $102 \pm 5.0$ & $7.7 \pm 0.3^{*}$ \\
DMEM control & $8.1 \pm 0.7 \times 10^{4}$ & $90 \pm 2.5$ & $8.2 \pm 0.5$ \\
$10 \mu$ g of anti-DARP-36aa & $5.0 \pm 1.0 \times 10^{4 * *}$ & $94 \pm 8.2$ & $5.1 \pm 0.4^{* *}$ \\
$50 \mu$ g of anti-DARP-36aa & $4.7 \pm 0.5 \times 10^{4 * *}$ & $93 \pm 2.1$ & $4.6 \pm 0.1^{* *}$ \\
\hline
\end{tabular}

Quantification of neuron number, cell body area, and number of neuronal branching points in primary mesencephalic cell cultures. Cultures treated with either $10 \mathrm{~nm}$ or $50 \mathrm{~nm}$ DARP-36aa were grown in serum-free N2 medium (control). Mesencephalic cells incubated with $10 \mu \mathrm{g}$ or $50 \mu \mathrm{g}$ of anti-DARP-36aa were maintained in serumsupplemented DMEM (control). Cultures were processed for NSE immunoreactivity on IVD 7 (DARP-36aa) or IVD 8 (anti-DARP-36aa) and analyzed by stereological methods (see Materials and Methods for details). Asterisks indicate groups that are significantly different from controls as follows: ${ }^{*} p<0.05 ;{ }^{* *} p<0.01$. Values were generated from two experiments with three separate culture wells examined per experiment. 
Statistical analysis. Statistical analysis was performed with a commercially available software package (SigmaStat 2.03; SPSS Science, Chicago, IL). The significance of differences between treatment groups was determined by one-way ANOVA. A Tukey test was used for pairwise multiple comparison procedures between treatment groups. In all cases a $p$ value of $\leq 0.05$ was considered significant.

\section{Results \\ DARP-36aa selectively promotes survival of mesencephalic neurons}

In this study, primary mesencephalic cell cultures were used to examine the ability of DARP-36aa to promote the survival of mesencephalic neurons. Mesencephalic cells were cultured for $7 \mathrm{~d}$ in chemically defined N2 medium. Cultures received 10 nм DARP-36aa, 50 nм DARP-36aa, or N2 medium alone and were processed for NSE immunoreactivity to assess the neuron number on IVD 7. Treatment with both 10 and 50 nM DARP-36aa resulted in a significant increase in mesencephalic neuron survival. Specifically, cultures that received 10 nM DARP-36aa had $\sim 85 \%$ more NSE-positive cells than untreated controls, whereas treatment with $50 \mathrm{~nm}$ DARP-36aa resulted in an approximate $70 \%$ increase in NSE-positive cells compared with control cultures (Table 1).

In addition to promoting neuron survival, DARP-36aa treatments resulted in increased morphological differentiation of NSE-immunoreactive cells in mesencephalic cultures (Fig. 1). Microscopic analysis revealed a striking increase in the number of branch points per neuron and cell connectivity in cultures treated with DARP-36aa. Control cultures (Fig. 1b) were found to have relatively low levels of connectivity and neurite branching compared with DARP-36aa-treated cultures (Fig. $1 c, d)$. However, incubation with DARP-36aa did not have an effect on cell soma size (Table 1). These findings demonstrate that DARP-36aa promotes the survival and morphological differentiation of mesencephalic neurons but does not affect cell soma area.

To further characterize the ability of DARP-36aa to promote the general survival and development of CNS cells, we investigated the effect of DARP-36aa on diencephalic and C6 glioma cell cultures. Figure $1 a$ compares the effect of DARP-36aa on mesencephalic neuron, diencephalic neuron, and C6 glioma cell number. Although DARP-36aa treatment resulted in a significant increase in the total number of mesencephalic neurons surviving on IVD 7, there was no significant effect of DARP-36aa on diencephalic neurons or C6 glioma cells. These results provide evidence for cell selectivity and specificity of DARP-36aa trophic activity.

\section{Anti-DARP-36aa selectively affects mesencephalic neuron survival and morphology}

Our laboratory has reported that in vivo immunoneutralization of DARP alters DA levels in the rat CNS (Llano and Ramirez,
1994). In an effort to determine whether in vitro immunoneutralization of endogenous DARP has an effect on neuron survival and development, we incubated primary mesencephalic cell cultures with a polyclonal antibody generated against DARP-36aa (antiDARP-36aa). Mesencephalic cells were cultured for $8 \mathrm{~d}$ in serum supplemented with DMEM and treated with $10 \mu \mathrm{g}$ of antiDARP-36aa, $50 \mu \mathrm{g}$ of anti-DARP-36aa, or serum-supplemented medium alone.

Mesencephalic cultures treated with $10 \mu \mathrm{g}$ of anti-DARP-36aa contained $43 \%$ fewer NSE-positive neurons than cultures grown in serum-supplemented DMEM alone. Treatment with $50 \mu \mathrm{g}$ of anti-DARP-36aa also resulted in a significant decrease in NSEpositive neurons on IVD 8 (Fig. 2, Table 1). Morphological analysis demonstrated that incubation with anti-DARP-36aa resulted in a dose-dependent decrease in neuronal branching and culture connectivity (Fig. $2 c, d$ ) versus controls (Fig. 2b). Treatment of mesencephalic cultures with anti-DARP-36aa did not significantly affect NSE-immunoreactive cell body area at either concentration examined (Table 1).

To determine whether other CNS cells respond to antiDARP-36aa, experiments similar to those performed for DARP36aa were conducted using diencephalic neurons and C6 glioma cells. Diencephalic neuron and C6 glioma cell survival and morphological development were not significantly affected by treatment with either $10 \mu \mathrm{g}$ of anti-DARP-36aa or $50 \mu \mathrm{g}$ of antiDARP-36aa (Fig. 2a). 


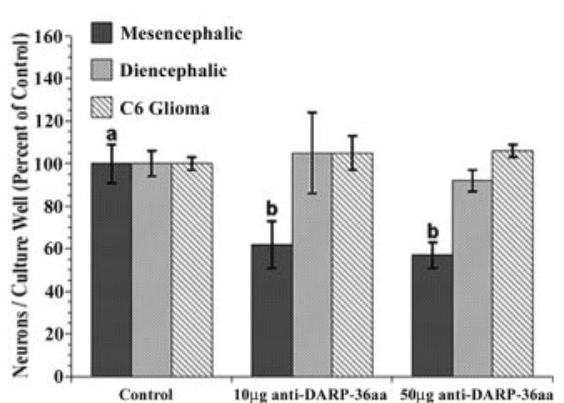

a
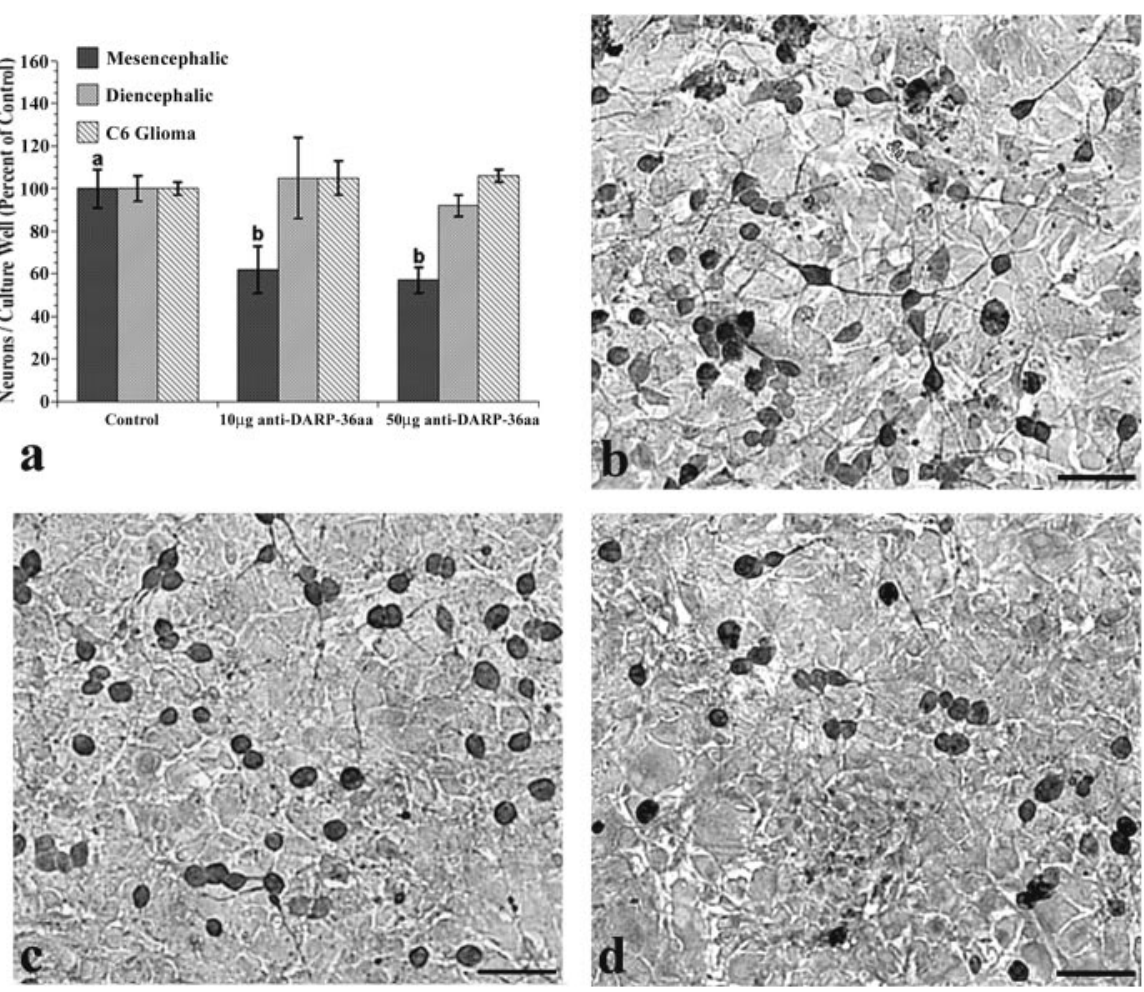

Figure 2. Evaluation of primary mesencephalic, diencephalic, and (6 glioma cell cultures after anti-DARP-36aa incubations. $a$, Treatment with anti-DARP-36aa resulted in a significant decrease in the total number of neurons (NSE-positive) surviving on culture day 8 in primary mesencephalic cultures. Cultures were grown for $8 \mathrm{~d}$ in serum-supplemented medium and treated with 10 $\mu \mathrm{g}$ of anti-DARP-36aa, $50 \mu \mathrm{g}$ of anti-DARP-36aa, or serum-supplemented medium alone (Control) on IVD 5 and IVD 7. Error bars indicate SEM; $n=6$. Different letters represent a significant decrease $(p<0.01)$ in neuron number between treatments. $b$, NSE-positive mesencephalic cells in serum-supplemented DMEM control cultures. Note the large number of NSE-positive cells and several branch points per neuron. c, Mesencephalic cultures treated with $10 \mu \mathrm{g}$ of anti-DARP-36aa had a marked decrease in NSE-positive cells and neuronal branch points. $d$, Treatment with $50 \mu \mathrm{g}$ of anti-DARP-36aa also resulted in a significant decrease in neuron number and decreased connectivity in mesencephalic cultures. Scale bars, $50 \mu \mathrm{m}$.

Table 2. Effects of DARP-36aa and anti-DARP-36aa on TH-positive neurons

\begin{tabular}{llcl}
\hline Treatment & $\begin{array}{l}\text { Neuron } \\
\text { number/well }\end{array}$ & $\begin{array}{l}\text { Cell body } \\
\text { area }\left(\mu \mathrm{m}^{2}\right)\end{array}$ & $\begin{array}{l}\text { Branching } \\
\text { points/neuron }\end{array}$ \\
\hline N2 control & $5.6 \pm 1.7 \times 10^{3}$ & $100 \pm 8.1$ & $2.9 \pm 0.3$ \\
$10 \mathrm{~nm}$ DARP-36aa & $1.8 \pm 0.2 \times 10^{4 *}$ & $108 \pm 6.7$ & $7.1 \pm 0.6^{*}$ \\
$50 \mathrm{~nm}$ DARP-36aa & $1.5 \pm 0.1 \times 10^{4 *}$ & $96 \pm 8.0$ & $7.5 \pm 0.8^{*}$ \\
DMEM control & $7.0 \pm 0.5 \times 10^{3}$ & $103 \pm 4.5$ & $5.9 \pm 0.5$ \\
$10 \mu \mathrm{g}$ of anti-DARP-36aa & $5.2 \pm 1.3 \times 10^{3}$ & $103 \pm 7.3$ & $2.8 \pm 0.3^{*}$ \\
$50 \mu \mathrm{g}$ of anti-DARP-36aa & $4.1 \pm 0.7 \times 10^{3 *}$ & $123 \pm 8.5$ & $2.9 \pm 0.4^{*}$
\end{tabular}

Quantification of TH-immunoreactive neuron number, cell body area, and number of neuronal branching points in primary mesencephalic cell cultures. Cultures treated with either $10 \mathrm{~nm}$ or $50 \mathrm{~nm}$ DARP-36aa were grown in serumfree N2 medium (control). Mesencephalic cells incubated with $10 \mu \mathrm{g}$ or $50 \mu \mathrm{g}$ of anti-DARP-36aa were maintained in serum-supplemented DMEM (control). Cultures were processed for TH immunoreactivity on IVD 7 (DARP-36aa) or IVD 8 (anti-DARP-36aa) and analyzed by stereological methods (see Materials and Methods for details). Asterisks indicate groups that are significantly different from controls as follows: ${ }^{*} p<0.01$. Values were generated from two experiments with three separate culture wells examined per experiment.

DARP-36aa increases catecholaminergic neuron number and morphological changes

We examined the effect that DARP-36aa has on catecholaminergic neuron survival in the mesencephalon by comparing the number of TH-immunoreactive cells in mesencephalic cultures. Immunocytochemical analysis of mesencephalic cultures revealed a relatively small population of TH-positive neurons in serum-free N2 control cultures on IVD 7. Cultures treated with DARP-36aa at concentrations of 10 and $50 \mathrm{~nm}$ showed a 2.5- to 3.2-fold increase in TH-positive neuron number compared with control cultures (Table 2). Based on the determination of TH-positive and total neuron num-

\section{Discussion}

bers, we estimated that the catecholaminergic neuron population represented $\sim 13,23$, and $21 \%$ of the total neuronal population in control, $10 \mathrm{~nm}$ DARP-36aa-treated, and $50 \mathrm{nM}$ DARP-36aa-treated cultures, respectively.

DARP-36aa-treated cultures revealed pronounced morphological changes of TH-immunoreactive neurons compared with N2 control cultures. Table 2 summarizes the quantification of $\mathrm{TH}$ immunoreactive neuron cell body area and branching points per neuron. Similar to NSE-immunoreactive neurons, DARP-36aa treatments significantly increased neuronal branching and had no significant effect on the average cell body area of TH-immunoreactive cells.

\section{Anti-DARP-36aa decreases catecholaminergic neuron number and morphological changes}

To expand further on the findings that DARP-36aa promotes the survival and development of catecholaminergic neurons in mesencephalic cell cultures, antiDARP-36aa was used in an attempt to immunoneutralize endogenous DARP in these cultures. Treatment with antiDARP-36aa resulted in a significant decrease in TH-immunoreactive neurons (Table 2, Fig. 3a). Incubating mesencephalic cultures with anti-DARP-36aa also resulted in a marked decrease in the number of branch points in the neuritic arborization of catecholaminergic neurons (Fig. 3b-f). Comparable with DARP-36aa incubations, anti-DARP-36aa treatments did not significantly affect TH-immunoreactive cell body area in mesencephalic cultures (Table 2).

\section{Genistein inhibits DARP-36aa induced neuron survival in primary mesencephalic cultures}

To examine the specificity of and to identify a putative signal transduction pathway for DARP-36aa, we examined the effect of genistein incubation on DARP-36aa-mediated neuron survival. A third independent experiment, similar to those described above for DARP-36aa, was performed to evaluate the effects of genistein incubations. As expected, incubation with $10 \mathrm{nM}$ DARP-36aa resulted in a significant increase in NSE-immunoreactive neuron survival on IVD 7 (Fig. 4a,d). Genistein treatments $(10 \mu \mathrm{g})$ did not significantly alter the number of NSE-immunoreactive neurons compared with EtOH control cultures (Fig. $4 a-c$ ). Interestingly, coincubation of $10 \mu \mathrm{g}$ of genistein with $10 \mathrm{~nm}$ DARP-36aa completely eliminated DARP-36aa-mediated increases in the number NSEimmunoreactive neurons per culture well (Fig. 4a,d,e).

Several well characterized factors provide trophic support to developing neuronal populations in the mesencephalon. Members of the neurotrophin family promote the survival and differentiation of dopaminergic and GABAergic neurons in the mesencephalon (Beck et al., 1993; Hyman et al., 1994; Ventimiglia et al., 
1995a; Loudes et al., 1999). GDNF is a potent regulator of survival and morphological differentiation of midbrain dopaminergic neurons and has also been shown to increase high-affinity dopamine uptake in these cells (Lin et al., 1993; Hou et al., 1996; Clarkson et al., 1997; Burke et al., 1998). Reports have shown that mitogenic growth factors, including basic fibroblast growth factor, insulin-like growth factor, and epidermal growth factor (EGF) promote survival and increase dopamine reuptake in cultured mesencephalic neurons (Ferrari et al., 1989, 1990; Knusel et al., 1990; Casper et al., 1994). In addition to the partial list of characterized trophic factors described above, there have been reports demonstrating the existence of several undefined trophic factors that promote the survival and development of mesencephalic neurons (Engele et al., 1996; Krieglstein and Unsicker, 1997; Panchision et al., 1998; Zhou et al., 2000).

In the present study, we assessed the effects of exogenously added DARP-36aa and immunoneutralization of DARP on the survival of mesencephalic neurons in vitro. Treatment of mesencephalic cultures with DARP-36aa resulted in a significant increase in neuron survival. Similar increases in the overall neuronal population have been observed in dissociated rat striatal cultures after brain-derived neurotrophic factor (BDNF) treatment (Ventimiglia et al., 1995a). However, DARP-36aa-mediated effects on NSE-immunoreactive neuron survival differ from the effects of other well characterized neurotrophic factors. Neurotrophin-3 (NT-3), neurotrophin-4/5 (NT-4/5), and BDNF (Hyman et al., 1994; Studer et al., 1995), as well as GDNF (Lin et al., 1993; Grondin and Gash, 1998), have survival-promoting effects on subpopulations of mesencephalic neurons but do not have a significant effect on overall mesencephalic neuronal population.

Mesencephalic cultures incubated with anti-DARP-36aa contained significantly fewer NSE-immunoreactive neurons compared with cultures grown with serum-supplemented medium alone. These findings correlate with and provide an explanation for previous reports that in vivo immunoneutralization of DARP resulted in a significant decrease in DA levels in the corpus striatum of postnatal rat pups (Kuhananthan et al., 1991). Also, it was found that E17 injections of anti-DARP induced fetal resorption and selectively altered mesencephalic DA concentration in treated animals (Llano and Ramirez, 1994). Similar decreases in neuronal survival have been observed after the incubation of blocking antibodies against NT-3 in hippocampal cultures (Lindholm et al., 1996) and in catecholaminergic caspase-3-activated DNase neuronal cell lines (Horton et al., 2001).

There has been considerable interest in the characterization of neurotrophic factors that regulate the survival and maintenance of dopaminergic neurons in the mesencephalon, given their rel- evance to several neurodegenerative diseases (Lindsay et al., 1993; Grondin and Gash, 1998). Therefore, we examined the effects of DARP-36aa and anti-DARP-36aa on the subpopulation of catecholaminergic neurons in the mesencephalon. Mesencephalic cultures maintained in the presence of DARP-36aa had a 3.2-fold increase in the number of $\mathrm{TH}$-immunoreactive neurons, whereas the immunoneutralization of DARP, from native DARPproducing cells (Choi et al., 1994), resulted in a 40\% decrease in TH-immunoreactive neurons compared with control cultures. The 3.2-fold increase in $\mathrm{TH}$-immunoreactive neuron number in DARP-36aa-treated cultures was considerably greater than the observed 1.8-fold increase in total neuron number in these cultures. These findings suggest that DARP-36aa treatments affect the development of catecholaminergic neurons as well as overall neuron survival. DARP-36aa-mediated increases in THimmunoreactive neuron number were similar to the effects of neurotrophins on dopaminergic neuron survival in mesencephalic cell cultures. BDNF treatments resulted in a threefold increase and NT-3 elicited a 2.3-fold increase in TH-positive neuron number on culture day 7 (Hyman et al., 1994). Interestingly, incubating mesencephalic cultures with $10 \mathrm{~nm}$ DARP-36aa elic- 

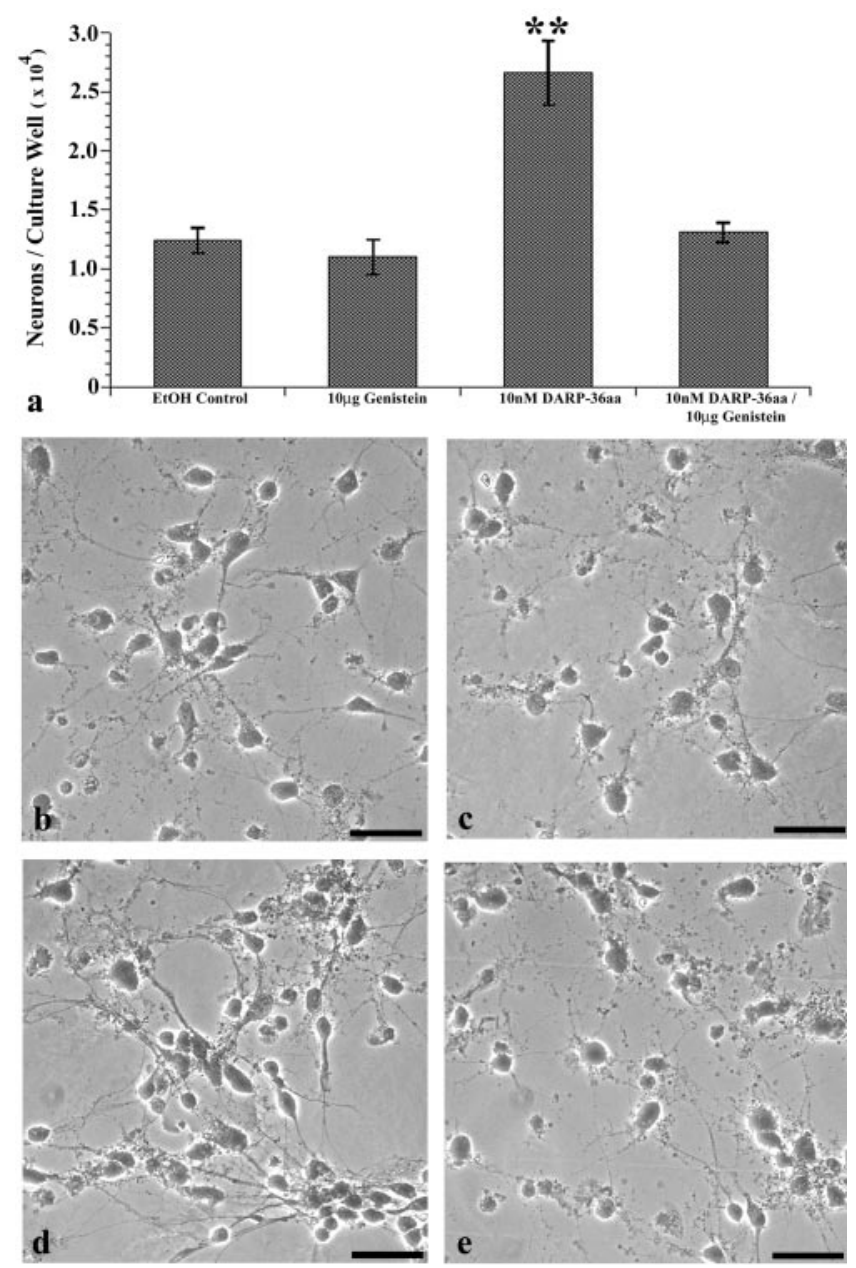

Figure 4. Genistein inhibits DARP-36aa-induced neuron survival in primary mesencephalic cultures on IVD 7. $a$, Treatment with $10 \mathrm{~nm}$ DARP-36aa resulted in a significant increase in the total number of neurons surviving on culture day 7. Genistein eliminated DARP-36aa-induced neuron survival, although it had no significant effect on neuron number when incubated in the absence of DARP-36aa. Cells were cultured for $7 \mathrm{~d}$ and treated with $10 \mathrm{~nm}$ DARP-36aa, $10 \mu \mathrm{g}$ of genistein, both $10 \mu \mathrm{g}$ of genistein and $10 \mathrm{~nm}$ DARP-36aa, or EtOH in serum-free N2 medium on IVD 2, IVD 4, and IVD 6. Asterisks indicate a significant difference $(p<0.01)$ in neuron number between treatment groups. Error bars indicate SEM. $b$, Phase contrast photomicrograph of primary mesencephalic cell cultures in $\mathrm{N} 2$ medium containing EtOH. c, Mesencephalic cultures treated with $10 \mu \mathrm{g}$ of genistein. $d$, Mesencephalic cultures have increased cell number and connectivity after incubation with $10 \mathrm{~nm}$ DARP-36aa.e, Incubation of $10 \mu \mathrm{g}$ of genistein with 10 nм DARP-36aa completely eliminated DARP-36aa-mediated increases in neuron number and culture connectivity. All photomicrographs were taken on IVD 7. Scale bars, $50 \mu \mathrm{m}$.

ited a greater increase in neuron number compared with $50 \mathrm{nM}$ DARP-36aa treatments. Hyman et al. (1994) have also reported that high concentrations of both NT-3 and NT-4/5 resulted in a slight attenuation of observed increases in $\mathrm{TH}$-immunoreactive cell number in mesencephalic cultures.

DARP was originally identified and isolated from the rat adrenal gland as a result of its ability to induce DA release from striatal tissue (Chang and Ramirez, 1988). Also, DARP was localized in and is released from rat C6 glioma cells (Smith and Ramirez, 1999). To address the possible effects of DARP-36aa on the survival of other distinct CNS cell populations, we examined the effect of DARP-36aa incubation in these physiologically relevant cells. In contrast to the pronounced effects of DARP-36aa and anti-DARP-36aa on mesencephalic neuron survival, neither DARP-36aa nor anti-DARP-36aa had any significant effect on diencephalic neuron survival. Neurotrophic factors have been shown to exert growth-promoting actions on distinct neuronal populations. Several studies have demonstrated that BDNF, NT-3, and NT-4/5 but not nerve growth factor regulate mesencephalic neuron survival (Knusel et al., 1990; Hyman et al., 1994). Also, BDNF, NT-3, and NT-4/5 have been shown to affect subpopulations of neurons differentially within the mesencephalon (Hyman et al., 1994; Ventimiglia et al., 1995a). The finding that DARP-36aa selectively promotes neuron survival in the mesencephalon but not in the diencephalon demonstrates that DARP36 aa does not regulate neuron survival in a general nonspecific manner. One might speculate that unaffected neurons did not express a putative DARP receptor, an important issue that requires additional research.

DARP-36aa and anti-DARP-36aa treatments had no significant effect on C6 glioma cell survival or proliferation at any concentration examined. In addition, very few non-neuronal cells were detected in mesencephalic cultures treated with DARP-36aa or in control cultures grown in serum-free N2 medium alone. These findings suggest that observed DARP-36aa-mediated neurotrophic effects occurred via a direct interaction with mesencephalic neurons and not through interaction with glial cells.

Morphological analysis revealed that DARP-36aa-treated neurons had a marked increase in branching points per NSEimmunoreactive neuron. Significant increases in neural branching were also observed after DARP-36aa treatments in $\mathrm{TH}$ positive neurons. Comparable increases in $\mathrm{TH}$-immunoreactive neuron branching have been observed in mesencephalic cultures treated with NT-4/5 (Studer et al., 1995). However, DARP-36aamediated increases in $\mathrm{TH}$-immunoreactive neuronal branching were not as robust as those reported for GDNF (Lin et al., 1993; Grondin and Gash, 1998). As expected, significant decreases in the number of branching points per neuron were observed in mesencephalic cultures after DARP immunoneutralization. In contrast to the neurotrophins and GDNF (Lin et al., 1993; Studer et al., 1995), DARP-36aa and anti-DARP-36aa treatments did not significantly alter the cell soma area.

The soy isoflavone genistein has been shown to attenuate the proliferative effects of several growth factors in vitro (Jing and Waxman, 1995; Peterson, 1995). We have reported recently that genistein significantly inhibits DARP-36aa internalization and transport in C6 glioma cells (Smith and Ramirez, 2002). In this study, we have shown that genistein completely attenuated DARP-36aa-mediated trophic effects in mesencephalic cell cultures. Genistein incubation in serum-free culture medium had no effect on neuron survival and morphological development, demonstrating that genistein selectively inhibits DARP-36aamediated trophic effects. Overall, these findings provide evidence for a putative receptor-signaling pathway for DARP-36aa. The antiproliferative effects of genistein are thought to be mediated through the inhibition of tyrosine protein kinase activity (Akiyama et al., 1987; Yang et al., 1996; Penar et al., 1997). Akiyama et al. (1987) reported that genistein is a highly specific inhibitor of tyrosine kinases but a relatively ineffective inhibitor of serine and threonine kinases as well as other ATP analogrelated enzymes. Several investigators have demonstrated that genistein is a specific inhibitor of tyrosine autophosphorylation of the EGF receptor (Akiyama et al., 1987; Peterson, 1995; Yang et al., 1996; Penar et al., 1997). Current findings, coupled with DARP-36aa internalization studies (Smith and Ramirez, 2002), suggest that DARP-36aa may be a ligand for the EGF receptor, or more likely, a yet uncharacterized receptor with related signaltransduction properties. However, recent studies in our laboratory have demonstrated that DARP-36aa does not activate 
mitogen-activated protein kinase pathways in cultured mesencephalic neurons (our unpublished observations).

The adrenal gland is a source for a large number of neurotrophically active neuropeptides. Adrenal chromaffin cells express BDNF, NT-4/5, GDNF, and TGF- $\beta$ (Unsicker, 1993; Krieglstein et al., 1996; Suter-Crazzolara et al., 1996). Interestingly, Krieglstein and Unsicker (1997) have reported the existence of a putative growth factor for mesencephalic dopaminergic neurons from adrenal chromaffin granules. DARP was originally purified from rat adrenal homogenates as a result of its ability to induce DA release from striatal tissue in vitro (Chang and Ramirez, 1988). Immunocytochemical studies have demonstrated the localization of DARP immunoreactivity in chromaffin granules of the rat adrenal medulla (Choi et al., 1993). Later, it was shown that immunoneutralization of DARP resulted in increased fetal resorption and selective alteration of DA concentrations in the rat mesencephalon (Kuhananthan et al., 1991; Llano and Ramirez, 1994). The present study reveals that DARP-36aa promotes neuron survival and development, whereas the immunoneutralization of DARP decreases the survival and development of mesencephalic neurons. Overall, the findings indicate that DARP is most likely a novel neurotrophic peptide for mesencephalic neurons produced by adrenal chromaffin and glial cells.

\section{References}

Akiyama T, Ishida J, Nakagawa S, Ogawara H, Watanabe S, Itoh N, Shibuya M, Fukami Y (1987) Genistein, a specific inhibitor of tyrosine-specific protein kinases. J Biol Chem 262:5592-5595.

Barde YA (1989) Trophic factors and neuronal survival. Neuron 2:1525-1534.

Beck KD, Knusel B, Hefti F (1993) The nature of the trophic action of brainderived neurotrophic factor, des(1-3)-insulin-like growth factor-1, and basic fibroblast growth factor on mesencephalic dopaminergic neurons developing in culture. Neuroscience 52:855-866.

Benda P, Lightbody J, Sato G, Levine L, Sweet W (1968) Differentiated rat glial cell strain in tissue culture. Science 161:370-371.

Bottenstein J, Hayashi I, Hutchings S, Masui H, Mather J, McClure DB, Ohasa S, Rizzino A, Sato G, Serrero G, Wolfe R, Wu R (1979) The growth of cells in serum-free hormone-supplemented media. Methods Enzymol 58:94-109.

Burke RE, Antonelli M, Sulzer D (1998) Glial cell line-derived neurotrophic growth factor inhibits apoptotic death of postnatal substantia nigra dopamine neurons in primary culture. J Neurochem 71:517-525.

Casper D, Roboz GJ, Blum M (1994) Epidermal growth factor and basic fibroblast growth factor have independent actions on mesencephalic dopamine neurons in culture. J Neurochem 62:2166-2177.

Chang GD, Ramirez VD (1988) A potent dopamine-releasing factor is present in high concentrations in the rat adrenal gland. Brain Res 463:385-389.

Choi WS, Kim MO, Ramirez VD (1993) Immunocytochemical localization of dopamine-releasing protein in the rat adrenal. Neuroendocrinology 58:440-447.

Choi WS, Lee BH, Ramirez VD (1994) Immunocytochemical mapping of a novel dopamine-releasing protein in the rat brain. Neuroendocrinology 60:194-204.

Clarkson ED, Zawada WM, Freed CR (1997) GDNF improves survival and reduces apoptosis in human embryonic dopaminergic neurons in vitro. Cell Tissue Res 289:207-210.

Engele J, Rieck H, Choi-Lundberg D, Bohn MC (1996) Evidence for a novel neurotrophic factor for dopaminergic neurons secreted from mesencephalic glial cell lines. J Neurosci Res 43:576-586.

Ferrari G, Minozzi MC, Toffano G, Leon A, Skaper SD (1989) Basic fibroblast growth factor promotes the survival and development of mesencephalic neurons in culture. Dev Biol 133:140-147.

Ferrari G, Minozzi MC, Toffano G, Leon A, Skaper SD (1990) Basic fibroblast growth factor affects the survival and development of mesencephalic neurons in culture. Adv Exp Med Biol 265:93-99.

Gibbs RB, Pfaff DW (1994) In situ hybridization detection of trkA mRNA in brain: distribution, colocalization with p75NGFR,and up-regulation by nerve growth factor. J Comp Neurol 341:324-339.

Grondin R, Gash DM (1998) Glial cell line-derived neurotrophic factor (GDNF): a drug candidate for the treatment of Parkinson's disease. J Neurol 245:35-42.

Horton CD, Qi Y, Chikaraishi D, Wang JK (2001) Neurotrophin-3 mediates the autocrine survival of the catecholaminergic CAD CNS neuronal cell line. J Neurochem 76:201-209.

Hou JG, Lin LF, Mytilineou C (1996) Glial cell line-derived neurotrophic factor exerts neurotrophic effects on dopaminergic neurons in vitro and promotes their survival and regrowth after damage by 1-methyl-4phenylpyridinium. J Neurochem 66:74-82.

Hyman C, Juhasz M, Jackson C, Wright P, Ip NY, Lindsay RM (1994) Overlapping and distinct actions of the neurotrophins BDNF, NT-3, and NT$4 / 5$ on cultured dopaminergic and GABAergic neurons of the ventral mesencephalon. J Neurosci 14:335-347.

Jing Y, Waxman S (1995) Structural requirements for differentiationinduction and growth-inhibition of mouse erythroleukemia cells by isoflavones. Anticancer Res 15:1147-1152.

Knusel B, Michel PP, Schwaber JS, Hefti F (1990) Selective and nonselective stimulation of central cholinergic and dopaminergic development in vitroby nerve growth factor, basic fibroblast growth factor, epidermal growth factor, insulin, and the insulin-like growth factors I and II. J Neurosci 10:558-570.

Kokaia Z, Bengzon J, Metsis M, Kokaia M, Persson H, Lindvall O (1993) Coexpression of neurotrophins and their receptors in neurons of the central nervous system. Proc Natl Acad Sci USA 90:6711-6715.

Korsching S (1993) The neurotrophic factor concept: a reexamination. J Neurosci 13:2739-2748.

Krieglstein K, Unsicker K (1997) Protein from chromaffin granules promotes survival of mesencephalic dopaminergic neurons by an EGFreceptor ligand-mediated mechanism. J Neurosci Res 48:18-30.

Krieglstein K, Deimling F, Suter-Crazzolara C, Unsicker K (1996) Expression and localization of GDNF in developing and adult adrenal chromaffin cells. Cell Tissue Res 286:263-268.

Kuhananthan S, Miklasz S, Ramirez VD (1991) Monoclonal antibodies against a dopamine-releasing protein (DARP) arrest fetal development, decrease brain catecholamines, and increase adrenal weight of neonatal rats. Mol Cell Neurosci 2:410-417.

Lapchak PA, Miller PJ, Jiao S, Araujo DM, Hilt D, Collins F (1996) Biology of glial cell line-derived neurotrophic factor (GDNF): implications for the use of GDNF to treat Parkinson's disease. Neurodegeneration 5:197-205.

Lin LF, Doherty DH, Lile JD, Bektesh S, Collins F (1993) GDNF: a glial cell line-derived neurotrophic factor for midbrain dopaminergic neurons. Science 260:1130-1132.

Lindholm D, Carroll P, Tzimagiogis G, Thoenen H (1996) Autocrineparacrine regulation of hippocampal neuron survival by IGF-1 and the neurotrophins BDNF, NT-3, and NT-4. Eur J Neurosci 8:1452-1460.

Lindsay RM, Altar CA, Cedarbaum JM, Hyman C, Wiegand SJ (1993) The therapeutic potential of neurotrophic factors in the treatment of Parkinson's disease. Exp Neurol 124:103-118.

Lindsay RM, Wiegand SJ, Altar CA, DiStefano PS (1994) Neurotrophic factors: from molecule to man. Trends Neurosci 17:182-190.

Llano DA, Ramirez VD (1994) Isolation of DARP (dopamine-releasing protein) from fetal rat brain and effects of DARP immunoneutralization on fetal mesencephalic dopamine levels. Mol Cell Neurosci 5:649-657.

Loudes C, Petit F, Kordon C, Faivre-Bauman A (1999) Distinct populations of hypothalamic dopaminergic neurons exhibit differential responses to brain-derived neurotrophic factor (BDNF) and neurotrophin-3 (NT3). Eur J Neurosci 11:617-624.

Mufson EJ, Kroin JS, Sendera TJ, Sobreviela T (1999) Distribution and retrograde transport of trophic factors in the central nervous system: functional implications for the treatment of neurodegenerative diseases. Prog Neurobiol 57:451-484.

Panchision DM, Martin-DeLeon PA, Takeshima T, Johnston JM, Shimoda K, Tsoulfas P, McKay RD, Commissiong JW (1998) An immortalized, type-1 astrocyte of mesencephalic origin source of a dopaminergic neurotrophic factor. J Mol Neurosci 11:209-221.

Penar PL, Khoshyomn S, Bhushan A, Tritton TR (1997) Inhibition of epidermal growth factor receptor-associated tyrosine kinase blocks glioblastoma invasion of the brain. Neurosurgery 40:141-151. 
Peterson G (1995) Evaluation of the biochemical targets of genistein in tumor cells. J Nutr 125:784S-789S.

Smith S, Ramirez VD (1999) Cellular localization of dopamine-releasing protein (DARP) in rat C6 glioma and primary mesencephalic cell cultures. Brain Res 843:95-104.

Smith S, Ramirez VD (2002) Direct visualization of internalization and intracellular trafficking of dopamine-releasing protein-36aa. Neuroendocrinology 75:98-112.

Snider WD (1994) Functions of the neurotrophins during nervous system development: what the knockouts are teaching us. Cell 77:627-638.

Studer L, Spenger C, Seiler RW, Altar CA, Lindsay RM, Hyman C (1995) Comparison of the effects of the neurotrophins on the morphological structure of dopaminergic neurons in cultures of rat substantia nigra. Eur J Neurosci 7:223-233.

Suter-Crazzolara C, Lachmund A, Arab SF, Unsicker K (1996) Expression of neurotrophins and their receptors in the developing and adult rat adrenal gland. Brain Res Mol Brain Res 43:351-355.
Unsicker K (1993) The trophic cocktail made by adrenal chromaffin cells. Exp Neurol 123:167-173.

Ventimiglia R, Mather PE, Jones BE, Lindsay RM (1995a) The neurotrophins BDNF, NT-3, and NT-4/5 promote survival and morphological and biochemical differentiation of striatal neurons in vitro. Eur J Neurosci 7:213-222.

Ventimiglia R, Jones BE, Moller A (1995b) A quantitative method for morphometric analysis in neuronal cell culture: unbiased estimation of neuron area and number of branch points. J Neurosci Methods 57:63-66.

Yang EB, Wang DF, Mack P, L. Y. C (1996) Genistein,a tyrosine kinase inhibitor, reduces EGF-induced EGF receptor internalization and degradation in human hepatoma HepG2 cells. Biochem Biophys Res Commun 224:309-317

Zhou J, Shen Y, Tang Z, Xu L, Bradford HF, Yu Y (2000) Striatal extracts promote the survival and phenotypic expression of rat fetal dopaminergic neurons in vitro. Neurosci Lett 292:5-8. 